\title{
Sur la structure de l'ensemble des points de discontinuité des fonctions qui admettent une dérivée aux points de continuité
}

\section{F. M. Filipczak (Lódź)}

Introduction. Dans ce travail nous nous occuperons seulement des fonctions réelles, finies, définies sur l'intervalle fermé $\langle 0,1\rangle=I$. Néanmoins, tous les lemmes et les théorèmes que nous y établissons restent en vigueur aussi dans le cas où les fonctions étudiées sont définies sur des intervalles arbitraires, finis ou non.

On sait que les conditions sous lesquelles: $1^{\circ}$ une fonction $f$ est continue au point $x, 2^{\circ}$ il existe une dérivée $f^{\prime}(x)$ finie, et $3^{\circ}$ il existe une dérivée $f^{\prime}(x)$ non nécessairement finie, ne sont pas équivalentes. Dans le travail [2] Kronrod a étudié la structure de l'ensemble des points de discontinuité des fonctions qui admettent une dérivée finie en tout point de continuité, c'est-à-dire des fonctions pour lesquelles les conditions $1^{\circ}$ et $2^{\circ}$ sont équivalentes. Le théorème établi par Kronrod peut être énoncé sous la forme suivante:

Pour que l'ensemble $E$ soit l'ensemble des points de discontinuité d'une fonction admettant une dérivée finie en tout point de continuité, il faut et il suffit que l'ensemble $E$ soit un $F_{\sigma}$ et en même temps un $G_{\delta}$.

Le but principal de ce travail est d'étudier la structure de l'ensemble des points de discontinuité des fonctions qui admettent une dérivée, finie ou non, en tout point de continuité. Cet ensemble sera caractérisé dans le théorème 2. Les lemmes 1-5 et 7 serviront à démontrer que la condition donnée dans ce théorème est nécessaire; les lemmes 5 et 6 , ainsi que le théorème 1 , prouveront qu'elle est suffisante.

Outre ce résultat, nous établirons le théorème 3 , dans lequel nous caractérisons l'ensemble des points de dérivabilité (c'est-à-dire des points où il existe une dérivée finie) d'une fonction admettant une dérivée (non nécessairement finie) en tout point de continuité.

Il est à remarquer que dans les théorèmes 2 et 3 la condition de l'existence d'une dérivée en tout point de continuité peut être remplacée par celle de l'existence d'une dérivée aux points, et seulement aux points 
qui sont points de continuité de la fonction, e'est-à-dire par une condition équivalente aux conditions $1^{0}$ et $3^{\circ}$ (en tout point $x$ ). Les démonstrations des théorèmes 2 et 3 ainsi modifiés restent les mêmes. par:

Notations. Soit $f$ une fonction réelle, finie, définie sur $I$. Désignons

$\underline{f}(x), \bar{f}(x), f^{-}(x), f^{+}(x)$ - respectivement la dérivée inférieure, supérieure, à gauche, à droite, de la fonction $f$ au point $x$;

$C_{f}, D_{f}, \Delta_{f}$ - respectivement l'ensemble des points de continuité, de discontinuité, de dérivabilité de la fonction f;

$\Delta_{f}^{*}$ - l'ensemble des points où il existe une dérivée (finie ou non) de la fonction $f$;

$P(x),(L(x))$ - l'ensemble des nombres limites à droite (à gauche) de la fonction $f$ au point $x$, c'est-ì-dire des nombres $y$ pour lesquels il existe une suite de points distincts $x_{n} \in I$ tels que

$$
x=\lim _{n \rightarrow \infty} x_{n}, \quad y=\lim _{n \rightarrow \infty} f\left(x_{n}\right) \quad \text { et } \quad x_{n}>x \quad\left(x_{n}<x\right) ;
$$

$A_{f}-$ l'ensemble des points de structure asymétrique de la fonction $\gamma$, c'est-à-dire des points tels que $L(x) \neq P(x)$;

$B_{f}-$ l'ensemble des points $x \in I$ pour lesquels $\underline{f}(x)=-\infty$ et $f(x)=\infty$, ainsi que $f(x) \in L(x)$ et $f(x) \in P(x)$;

ft - l'ensemble des fonctions $f$ telles que pour tout $x \in O_{f}$ on a $\underline{f}(x)>-\infty$ ou $\bar{f}(x)<\infty$.

Nous admettrons toujours dans la suite que les ensembles considérés sont contenus dans $I$.

\section{§ 1. Quelques propriétés des ensembles $F_{\sigma}$ et $G_{\delta}$ dans}

l'espace $I_{0}$. Soit $B \subset I$ un ensemble dénombrable et $I_{0}=I \backslash B$. L'ensemble $I_{0}$ est un $G_{\delta}$. On peut le considérer comme un espace séparable. Par fermeture (relativement a $I_{0}$ ) du sous-ensemble $\mathbb{E}$ de l'espace $I_{0}$ nous entendrons l'ensemble $\bar{E} \cap I_{0}$, où $\bar{E}$ désigne la fermeture de l'ensemble $E$ (dans $I$ ).

On démontre aisément que:

$\left(1^{\circ}\right) L^{\prime}$ ensemble $E_{0} \subset I_{0}$ est fermé, ouvert, $I_{\alpha}^{\prime}$ ou $G_{\delta}$ dans l'espace $I_{0}$ si et seulement si $E_{0}=E \cap I_{0}$, oì $E$ est un ensemble fermé, ouvert (relativement da $I), F_{\sigma}$ ou $G_{\delta}$.

$\left(2^{\circ}\right) \quad L^{\prime}$ 'ensemble $P$ fermé dans $I_{0}$ est parfait dans l'espace $I_{0}$ si et seulement si l'ensemble $\bar{P}$ est parfait.

Les théorèmes $\mathrm{V}$, p. 67 , et 5, p. 269, de la monographie de Kuratowski [3] entraínent le théorème suivant: $\left(3^{\circ}\right)$ Si dans un espace séparable on a $E \notin F_{\sigma}$ ou bien $E \notin G_{\delta}$, il existe un ensemble fermé $F \neq 0$ de cet espace tel que $F=\bar{F} \cap \bar{B} \cap \bar{F} \backslash \bar{E}$.

Remarque. La condition $F=\overline{F \cap E} \cap \overline{F \backslash} \backslash E$ est équivalente à l'égalité $F=\overline{F \cap E}=\overline{F \backslash E}$. L'ensemble $F$ satisfaisant à cette condition doit être parfait. En effet, si $x \in F$, on a $x \in \overline{F \cap E}$ et $x \in \overline{F \backslash E}$. Lorsque $x \in F \cap E$, on a évidemment $x \notin F \backslash E$ et, comme $x \in \overline{F \backslash E}$, $x$ est un point d'accumulation de l'ensemble $F \backslash E$, donc aussi de l'ensemble $F$. De façon analogue, si $x \in F \backslash E$, on a $x \notin F \cap E$ mais, comme $x \in \overline{F \cap E}$, $x$ est un point d'accumulation de l'ensemble $F \cap E \subset F$.

Du théorème $\left(3^{\circ}\right)$ et de la remarque précédente on déduit:

$\left(4^{\circ}\right)$ Si dans un espace séparable l'ensemble $E \notin F_{\sigma}$ ou bien $E \notin G_{\delta}^{*}$, il existe un ensemble parfait non vide $P$ de cet espace tel que $P=\overline{P \cap E}$ $=\overline{P \backslash E}$.

LEMLME 1. Si $\mathbb{E}=A \cup B$, ò̀ $A \cap B=0, A \in G_{\delta}, B$ est un ensemble dénombrable, et si pour tout ensemble parfait $P$ la condition: „E $D \cap P$ est un ensemble frontière dans $P$ " entraîne la condition: "E $\cap P$ est de première catégorie de Baire dans $P^{\prime \prime}$, alors $E$ est un $F_{\sigma}$.

Démonstration. Supposons que l'ensemble $A$ ne soit pas un $F_{\sigma}$ dans l'ensemble $I_{0}=I \backslash B$. En vertu de $\left(4^{\circ}\right)$ il existe un ensemble $P_{0} \subset I_{0}$ parfait dans $I_{0}$ et tel que l'ensemble $P_{0} \cap A$ est dense et en même temps ensemble frontière dans $P_{0}$. Soit $P=\bar{P}_{0}$, donc $P$ est un ensemble parfait et $P_{0}=P \cap I_{0}$. L'ensemble $E \cap P$ est un ensemble frontière dans $P$. En effet, si $K$ est une portion de l'ensemble $P$, c'est-à-dire un ensemble parfait non vide de la forme $\langle a, b\rangle \cap P$, l'ensemble $K_{0}=K \cap I_{0}$ est une portion de l'ensemble $P_{0}$. L'ensemble $A \cap P_{0}$ étant un ensemble frontière dans $P_{0}$, il existe un point $x \in \Pi_{0} \backslash A$. Évidemment $x \notin B$, done $x \notin A \cup B=E$. Nous avons ainsi obtenu $x \in K \backslash E$. L'ensemble $P \cap E$ est donc un ensemble frontière dans $P$. De là et de l'hypothèse du lemme il résulte que l'ensemble $P \cap E$ est de première catégorie dans $P$.

D'autre part, l'ensemble $P_{0} \cap A$ est dense dans $P$, ear il est dense dans $P_{0}$. Etant un $G_{\delta}$, il est résidual dans $P$. A fortiori l'ensemble $P \cap E$ est résiduel dans $P$. Cette conclusion est incompatible avec la précédente, en vertu de laquelle l'ensemble $P \cap E$ est de première catégorie dans $P$. Cette contradiction prouve que l'ensemble $A$ est un $F_{\sigma}$ dans l'espace $I_{0}$, on a donc, en vertiu de $\left(1^{\circ}\right), A=A^{\prime} \cap I_{0}$, où $A^{\prime} \in F_{\sigma} . A=A^{\prime} \cap(I \backslash B)$ $=A^{\prime} \backslash B, E=A \cup B=\left(A^{\prime} \backslash B\right) \cup B=A^{\prime} \cup B$. De cette dernière égalité il résulte que $E \in F_{\sigma}$, c.q.f.d.

Le lemme suivant est dual au précédent.

Lenave 2. Si $E=A \backslash B$, où $A \in F_{\sigma}, B$ est un ensemble dénombrable, et si pour tout ensemble parfait $P$ l'hypothèse: , $P \cap \mathbb{E}$ est dense dans $P$ " entraîne: "P $P \cap E$ est résiduel dans $P "$, alors $E$ est un $G_{\delta}$. 


\section{\$2. La classe borelienne de l'ensemble $B_{f}$.}

LeMre 3. Si $f \in \mathcal{H}$, on a $B_{f}=D_{f} \backslash E$, oì $E$ est un ensemble dénombrable.

Démonstration. Désignons par $A$ l'ensemble des points $x$ tels que $f(x) \notin L(x)$ ou bien $f(x) \notin P(x)$. On sait que les ensembles $A$ et $A_{f}$ sont denombrables (v. [6] et [9]). On voit aisément que si $f \epsilon \mathfrak{t t}$, on a $D_{f} \backslash\left(A \cup A_{f}\right) \subset B_{f} \subset D_{f}$. Des deux conclusions précédentes résulte notre lemme.

LeMvas 4. Si $f \in \mathcal{A}$, on a $B_{f} \in G_{\delta}$.

Démonstration. On sait que l'ensemble $D_{f}$ est un $F_{\sigma}$ (voir p.ex. [3], p. 102). En tenant compte des lemmes 2 et 3 il suffit de prouver que pour tout ensemble parfait $P$ l'hypothèse: "l'ensemble $P \cap B_{f}$ est dense dans $P$ " entraîne: ,l'ensemble $P \cap B_{f}$ est résiduel dans $P$ ". Supposons le contraire et admettons qu'il existe un ensemble parfait $P_{0} \neq 0$ tel que l'ensemble $P_{0} \cap B_{f}$ est dense, mais n'est pas résiduel dans $P_{0}$.

En vertu du lemme 3 l'ensemble $P_{0} \cap B_{f}$ est un $F_{\sigma \delta}$, il satisfait donc à la condition de Baire. Avec la conclusion précédente cela prouve qu'il existe une portion $P$ de l'ensemble $P_{0}$, sur laquelle l'ensemble $P \cap B_{f}$ est de première catégorie de Baire. L'ensemble $P \cap D_{f}$ est aussi de première catégorie dans $P$, puisque, en vertu du lemme 2, l'ensemble $D_{f} \backslash B_{f}$ est dénombrable.

L'ensemble $P \cap C_{f}$ est un $G_{\delta}$, donc $P \cap O_{f}=\bigcap_{n=1}^{\infty} G_{n}$, où les $G_{n}$ sont des ensembles ouverts et $G_{n} \supset G_{n+1}$, pour $n=1,2, \ldots$

Soit $a=\min _{t \in P} t, b=\max _{t \in P} t$. Nous définirons maintenant les suites de points $\left\{a_{n}\right\},\left\{b_{n}\right\},\left\{c_{n}\right\}$ et $\left\{d_{n}\right\}$, ainsi qu'une suite d'intervalles fermés $\left\{\left\langle r_{n}, s_{n}\right\rangle\right\}$, telles que soient vérifiées les conditions:

(A $\left.\mathrm{A}_{n}\right) \quad p_{n}<r_{n}<s_{n}<q_{n}, \quad$ où $\quad p_{n}=\max \left(a_{n}, c_{n}\right), q_{n}=\min \left(b_{n}, d_{n}\right)$,

$$
\begin{gathered}
0<b_{n}-a_{n}<\frac{1}{n}, \quad 0<d_{n}-c_{n}<\frac{1}{n}, \\
\left\langle r_{n}, s_{n}\right\rangle \subset \dot{G}_{n}, \\
\left(r_{n}, s_{n}\right) \cap P \neq 0, \\
\frac{f\left(b_{n}\right)-f\left(a_{n}\right)}{b_{n}-a_{n}}>n, \quad \frac{f\left(d_{n}\right)-f\left(c_{n}\right)}{d_{n}-c_{n}}<-n, \\
\left\langle r_{n}, s_{n}\right\rangle \subset\left(r_{n-1}, s_{n-1}\right), \quad\left(\left\langle r_{1}, s_{1}\right\rangle \subset(a, b)\right) .
\end{gathered}
$$

Soit $\xi \neq a$ et $\xi \neq b$ un point quelconque de l'ensemble $P \cap B_{f}$. Un tel point existe, car l'ensemble $P \cap B_{f}$ est dense dans $P$. La condition $\xi \in B_{f}$ signifie que $\bar{f}(\xi)=\infty, \underline{f}(\xi)=-\infty, f(\xi) \in L(\xi)$ et $f(\xi) \in P(\xi)$. Il en résulte qu'il existe des points $\alpha_{1}$ et $\gamma_{1}$ de l'intervalle $(a, b)$ et des suites $\left\{\xi_{k}\right\}$ et $\left\{\eta_{k}\right\}$ tels que les conditions suivantes sont remplies:

(3) $\operatorname{sgn}\left(\xi_{k}-\xi\right)=\operatorname{sgn}\left(\xi-a_{1}\right) \quad$ pour $k=1,2, \ldots, \lim _{k \rightarrow \infty} \xi_{k}=\xi$,

$$
\lim _{k \rightarrow \infty} f\left(\xi_{k}\right)=f(\xi)
$$

(4) $\operatorname{sgn}\left(\eta_{k}-\xi\right)=\operatorname{sgn}\left(\xi-\gamma_{1}\right) \quad$ pour $k=1,2, \ldots, \lim _{k \rightarrow \infty} \eta_{k}=\xi$,

$$
\lim _{k \rightarrow \infty} f(\eta k)=f(\xi) \text {. }
$$

Des deux dernières égalités (3) il résulte que dans l'intervalle $\left(\xi, 2 \xi-\alpha_{1}\right)\left({ }^{1}\right)$ il existe un point $\xi_{k} \epsilon(a, b)$, que nous désignerons par $\beta_{1}$ et qui satisfait à l'inégalité

$$
\left|f\left(\beta_{1}\right)-f(\xi)\right|<\frac{1}{4}\left|f(\xi)-f\left(\alpha_{1}\right)\right| .
$$

D'une manière analogue on déduit des égalités (4) que dans l'intervalle $\left(\xi, 2 \xi-\gamma_{1}\right)$ il existe parmi les points $\eta_{k} \epsilon(a, b)$ un point $\delta_{1}$ tel que

$$
\left|f\left(\delta_{1}\right)-f(\xi)\right|<\frac{1}{4}\left|f(\xi)-f\left(\gamma_{1}\right)\right| \text {. }
$$

Nous établirons plus loin les relations suivantes:

$$
\begin{aligned}
\frac{f\left(\beta_{1}\right)-f\left(\alpha_{1}\right)}{\beta_{1}-\alpha_{1}} & =\frac{f\left(\beta_{1}\right)-f(\xi)}{\beta_{1}-\alpha_{1}}+\frac{f(\xi)-f\left(\alpha_{1}\right)}{\beta_{1}-\alpha_{1}} \\
& \geqslant \frac{f(\xi)-f\left(\alpha_{1}\right)}{\beta_{1}-\xi+\xi-\alpha_{1}}-\left|\frac{f\left(\beta_{1}\right)-f(\xi)}{\beta_{1}-\xi+\xi-\alpha_{1}}\right| \\
& >\frac{f(\xi)-f\left(\alpha_{1}\right)}{2\left(\xi-\alpha_{1}\right)}-\frac{1}{4} \cdot \frac{\left|f(\xi)-f\left(\alpha_{1}\right)\right|}{\left|\xi-\alpha_{1}\right|}=\frac{1}{4} \cdot \frac{f(\xi)-f\left(\alpha_{1}\right)}{\xi-a_{1}}>1
\end{aligned}
$$

(10) $\frac{f\left(\delta_{1}\right)-f\left(\gamma_{1}\right)}{\delta_{1}-\gamma_{1}}=\frac{f\left(\delta_{1}\right)-f(\xi)}{\delta_{1}-\gamma_{1}}+\frac{f(\xi)-f\left(\gamma_{1}\right)}{\delta_{1}-\gamma_{1}}$

$$
\begin{aligned}
& \leqslant \frac{f(\xi)-f\left(\gamma_{1}\right)}{\delta_{1}-\xi+\xi-\gamma_{1}}+\left|\frac{f\left(\delta_{1}\right)-f(\xi)}{\delta_{1}-\xi+\xi-\gamma_{1}}\right| \\
& <\frac{f(\xi)-f\left(\gamma_{1}\right)}{2\left(\xi-\gamma_{1}\right)}+\frac{1}{4} \cdot \frac{\left|f(\xi)-f\left(\gamma_{1}\right)\right|}{\left|\xi-\gamma_{1}\right|}=\frac{1}{4} \cdot \frac{f(\xi)-f\left(\gamma_{1}\right)}{\xi-\gamma_{1}}<-1 .
\end{aligned}
$$

() Si $x>y,(x, y)$ désigne l'intervalle $(y, x)$. 
Les égalités (7) résultent des définitions des points $\beta_{1}$ et $\delta_{1}$, ainsi que des égalités (3) et (4); les relations (8) s'obtiennent de la condition $\beta_{1} \epsilon\left(\xi, 2 \xi-\alpha_{1}\right)$ et de (1), ainsi que de la condition $\delta_{1} \in\left(\xi, 2 \xi-\gamma_{1}\right)$ et de (2), enfin les relations (9) et (10) sont des conséquences des conditions (7), $(8),(5)$ et (1) ainsi que des relations $(7),(8),(6)$ et (2).

Posons

$$
\begin{array}{ll}
a_{1}=\min \left(\alpha_{1}, \beta_{1}\right), & b_{1}=\max \left(\alpha_{1}, \beta_{1}\right), \\
c_{1}=\min \left(\gamma_{1}, \delta_{1}\right), & \dot{d}_{1}=\max \left(\gamma_{1}, \delta_{1}\right) .
\end{array}
$$

On a évidemment les inégalités $\left(\mathrm{B}_{1}\right)$ et les suivantes:

$$
a<a_{1}<\xi<b_{1}<b, \quad a<c_{1}<\xi<d_{1}<b .
$$

De (9) et (10) il s'ensuit que les conditions $\left(\mathbb{E}_{1}\right)$ sont aussi remplies. L'ensemble $\left(p_{1}, q_{1}\right) \cap P \neq 0$, puisque, en vertu de (11), $\xi \in\left(p_{1}, q_{1}\right)$. Nous avons aussi prouvé que $P \cap D_{f}$ est de première catégorie dans $P$, il existe done dans l'ensemble $\left(p_{1}, q_{1}\right) \cap P$ un point $\eta \in P \cap C_{f} \subset G_{1}$. Désignons par $\left(r_{1}, s_{1}\right)$ un intervalle quelconque contenant le point $\eta$ et tel que $\left\langle r_{1}, s_{1}\right\rangle \subset G_{1} \cap\left(p_{1}, q_{1}\right)$. De cette inclusion résulte $\left(\mathrm{A}_{1}\right)$. Les conditions $\left(\mathrm{C}_{1}\right)$ et $\left(\mathrm{D}_{1}\right)$ sont évidemment remplies. En outre, on a $\left\langle r_{1}, s_{1}\right\rangle \subset(a, b)$.

Considérons des points déterminés $a_{n}, b_{n}, c_{n}$ et $d_{n}$ et un intervalle $\left\langle i_{n}, s_{n}\right\rangle$ tels que les conditions $\left(\mathbf{A}_{n}\right)-\left(\mathbf{F}_{n}\right)$ soient remplies. Dans l'ensemble $\left(r_{n}, s_{n}\right) \cap P \neq 0$ il existe un point $\xi^{\prime} \in P \cap B_{f}$, il existe donc aussi des points $\alpha_{n+1}$ et $\gamma_{n+1}$ appartenant à l'intervalle $\left(r_{n}, s_{n}\right)$ et des suites $\left\{\xi_{k}^{\prime}\right\}$ et $\left\{\eta_{k}^{\prime}\right\}$ satisfaisant aux conditions suivantes:

$$
\begin{array}{ll}
\left|\xi^{\prime}-a_{n+1}\right|<\frac{1}{2(n+1)}, & \frac{f\left(\xi^{\prime}\right)-f\left(a_{n+1}\right)}{\xi^{\prime}-a_{n+1}}>4(n+1), \\
\left|\xi^{\prime}-\gamma_{n+1}\right|<\frac{1}{2(n+1)}, & \frac{f\left(\xi^{\prime}\right)-f\left(\gamma_{n+1}\right)}{\xi^{\prime}-\gamma_{n+1}}<-4(n+1),
\end{array}
$$

(3') $\operatorname{sgn}\left(\xi_{k}^{\prime}-\xi^{\prime}\right)=\operatorname{sgn}\left(\xi^{\prime}-a_{n+1}\right) \quad$ pour $k=1,2, \ldots, \lim _{k \rightarrow \infty} \xi_{k}^{\prime}=\xi^{\prime}$,

$$
\lim _{k \rightarrow \infty} f\left(\xi_{k}^{\prime}\right)=f\left(\xi^{\prime}\right)
$$

$\left(4^{\prime}\right) \quad \operatorname{sgn}\left(\eta_{k}^{\prime}-\xi^{\prime}\right)=\operatorname{sgn}\left(\xi^{\prime}-\gamma_{n+1}\right) \quad$ pour $k=1,2, \ldots, \lim _{k \rightarrow \infty} \eta_{k}^{\prime}=\xi^{\prime}$,

$$
\lim _{k \rightarrow \infty} f\left(\eta_{\eta_{k}}^{\prime}\right)=f\left(\xi^{\prime}\right) .
$$

Des relations $\left(3^{\prime}\right)$ et $\left(4^{\prime}\right)$ on conclut que dans les intervalles $\left(\xi^{\prime}, 2 \xi^{\prime}-a_{n+1}\right)$ et $\left(\xi^{\prime}, 2 \xi^{\prime}-\gamma_{n+1}\right)$ il existe respectivement des points $\xi_{m}^{\prime}$ et $\eta_{n}^{\prime}$ de l'intervalle $\left(r_{n}, s_{n}\right)$, que nous désignerons par $\beta_{n+1}$ et $\delta_{n+1}$ et qui satisfont aux conditions

$$
\begin{aligned}
& \left|f\left(\beta_{n+1}\right)-f\left(\xi^{\prime}\right)\right|<1\left|f\left(\xi^{\prime}\right)-f\left(\alpha_{n+1}\right)\right|, \\
& \left|f\left(\delta_{n+1}\right)-f\left(\xi^{\prime}\right)\right|<\frac{1}{4}\left|f\left(\xi^{\prime}\right)-f\left(\gamma_{n+1}\right)\right| .
\end{aligned}
$$

Observons maintenant que l'on a les relations suivantes:

$\left(7^{\prime}\right) \operatorname{sgn}\left(\beta_{n+1}-\xi^{\prime}\right)=\operatorname{sgn}\left(\xi^{\prime}-\alpha_{n+1}\right), \quad \operatorname{sgn}\left(\delta_{n+1}-\xi^{\prime}\right)=\operatorname{sgn}\left(\xi^{\prime}-\gamma_{n+1}\right)$,

(8) $\left|\beta_{n+1}-\xi^{\prime}\right|<\left|\xi^{\prime}-\alpha_{n+1}\right|<\frac{1}{2(n+1)}, \quad\left|\delta_{n+1}-\xi^{\prime}\right|<\left|\xi^{\prime}-\gamma_{n+1}\right|<\frac{1}{2(n+1)}$,

$$
\begin{aligned}
\left(9^{\prime}\right) \quad \frac{f\left(\beta_{n+1}\right)-f\left(a_{n+1}\right)}{\beta_{n+1}-a_{n+1}} & =\frac{f\left(\beta_{n+1}\right)-f\left(\xi^{\prime}\right)}{\beta_{n+1}-a_{n+1}}+\frac{f\left(\xi^{\prime}\right)-f\left(a_{n+1}\right)}{\beta_{n+1}-a_{n+1}} \\
& \geqslant \frac{f\left(\xi^{\prime}\right)-f\left(\alpha_{n+1}\right)}{\beta_{n+1}-\xi^{\prime}+\xi^{\prime}-a_{n+1}}-\left|\frac{f\left(\beta_{n+1}\right)-f\left(\xi^{\prime}\right)}{\beta_{n+1}-\xi^{\prime}+\xi^{\prime}-a_{n+1}}\right| \\
& >\frac{f\left(\xi^{\prime}\right)-f\left(a_{n+1}\right)}{2\left(\xi^{\prime}-a_{n+1}\right)}-\frac{1}{4} \cdot \frac{\left|f\left(\xi^{\prime}\right)-f\left(a_{n+1}\right)\right|}{\left|\xi^{\prime}-a_{n+1}\right|} \\
& =\frac{1}{4} \cdot \frac{f\left(\xi^{\prime}\right)-f\left(\alpha_{n+1}\right)}{\xi^{\prime}-\alpha_{n+1}}>n+1, \\
\left(10^{\prime}\right) \quad \frac{f\left(\delta_{n+1}\right)-f\left(\gamma_{n+1}\right)}{\delta_{n+1}-\gamma_{n+1}} & =\frac{f\left(\delta_{n+1}\right)-f\left(\xi^{\prime}\right)}{\delta_{n+1}-\gamma_{n+1}}+\frac{f\left(\xi^{\prime}\right)-f\left(\gamma_{n+1}\right)}{\delta_{n+1}-\gamma_{n+1}} \\
& \leqslant \frac{f\left(\xi^{\prime}\right)-f\left(\gamma_{n+1}\right)}{\delta_{n+1}-\xi^{\prime}+\xi^{\prime}-\gamma_{n+1}}+\left|\frac{f\left(\delta_{n+1}\right)-f\left(\xi^{\prime}\right)}{\delta_{n+1}-\xi^{\prime}+\xi^{\prime}-\gamma_{n+1}}\right| \\
& <\frac{f\left(\xi^{\prime}\right)-f\left(\gamma_{n+1}\right)}{2\left(\xi^{\prime}-\gamma_{n+1}\right)}+\frac{1}{4} \cdot \frac{\left|f\left(\xi^{\prime}\right)-f\left(\gamma_{n+1}\right)\right|}{\left|\xi^{\prime}-\gamma_{n+1}\right|} \\
& =\frac{1}{4} \cdot \frac{f\left(\xi^{\prime}\right)-f\left(\gamma_{n+1}\right)}{\xi^{\prime}-\gamma_{n+1}}<-(n+1) .
\end{aligned}
$$

Afin d'établir les relations $\left(7^{\prime}\right)-\left(10^{\prime}\right)$, il suffit de répéter le raisonnement par lequel on a démontré les relations (7)-(10), en y remplaçant les relations (1)-(8) par $\left(1^{\prime}\right)-\left(8^{\prime}\right)$.

Posons

$$
\begin{array}{ll}
a_{n+1}=\min \left(\alpha_{n+1}, \beta_{n+1}\right), & b_{n+1}=\max \left(a_{n+1}, \beta_{n+1}\right), \\
c_{n+1}=\min \left(\gamma_{n+1}, \delta_{n+1}\right), & d_{n+1}=\max \left(\gamma_{n+1}, \delta_{n+1}\right) .
\end{array}
$$

Nous avons done, en tenant compte de $\left(7^{\prime}\right)$ et de $\alpha_{n+1} \neq \xi^{\prime} \neq \gamma_{n+1}$,

$$
\text { (11') } \quad r_{n}<a_{n+1}<\xi^{\prime}<b_{n+1}<s_{n}, \quad r_{n}<c_{n+1}<\xi^{\prime}<d_{n+1}<s_{n} .
$$

Les conditions $\left(\mathrm{B}_{n+1}\right)$ et $\left(\mathbb{B}_{n+1}\right)$ sont remplies. Cela résulte respectivement des inégalités $\left(1^{\prime}\right),\left(2^{\prime}\right)$ et $\left(8^{\prime}\right)$, ainsi que $\left(9^{\prime}\right)$ et $\left(10^{\prime}\right)$. De l'inégalité $\left(11^{\prime}\right)$ il résulte que l'ensemble $\left(p_{n+1}, q_{n+1}\right) \cap P \neq 0$, puisque le point $\xi^{\prime}$ est un élément de cet ensemble. L'ensemble $P \cap C_{f}$ étant dense dans $P$, il existe un point $\eta^{\prime} \in\left(p_{n+1}, q_{n+1}\right) \cap P \cap C_{f} \subset G_{n+1}$. Désignons par $\left(r_{n+1}, s_{n+1}\right)$ un intervalle quelconque contenant le point $\eta^{\prime}$ et tel que

$$
\left\langle r_{n+1}, s_{n+1}\right\rangle \subset G_{n+1} \cap\left(p_{n+1}, q_{n+1}\right) .
$$

Fundamenta Mathematicae, $T . X I$ 
Les considérations précédentes montrent que les conditions $\left(\mathrm{A}_{n+1}\right),\left(\mathrm{C}_{n+1}\right)$, $\left(\mathrm{D}_{n+1}\right)$ et, en vertu de $\left(11^{\prime}\right)$, aussi la condition $\left(\mathrm{F}_{n+1}\right)$ sont remplies. Posons

$$
\langle x\rangle=\langle x, x\rangle=\bigcap_{n=1}^{\infty}\left\langle r_{n}, s_{n}\right\rangle .
$$

Évidemment pour tout $n$ on a $a_{n}<x<b_{n}$ et $c_{n}<x<d_{n}$. Désignons maintenant par $x_{n}$ celui des points $a_{n}$ et $b_{n}$, et par $y_{n}$ celui des points $c_{n}$ et $d_{n}$ pour lesquels ont lieu les égalités

$$
\begin{aligned}
& \frac{f(x)-f\left(x_{n}\right)}{x-x_{n}}=\max \left(\frac{f(x)-f\left(a_{n}\right)}{x-a_{n}}, \frac{f(x)-f\left(b_{n}\right)}{x-b_{n}}\right), \\
& \frac{f(x)-f\left(y_{n}\right)}{x-y_{n}}=\min \left(\frac{f(x)-f\left(a_{n}\right)}{x-c_{n}}, \frac{f(x)-f\left(d_{n}\right)}{x-d_{n}}\right) .
\end{aligned}
$$

On a

$$
\begin{aligned}
\frac{f(x)-f\left(x_{n}\right)}{x-x_{n}} & =\max \left(\frac{f\left(b_{n}\right)-f(x)}{b_{n}-x}, \frac{f(x)-f\left(a_{n}\right)}{x-a_{n}}\right) \\
& \geqslant \frac{f\left(b_{n}\right)-f(x)+f(x)-f\left(a_{n}\right)}{b_{n}-x+x-a_{n}}>n,\left({ }^{2}\right) \\
\frac{f(x)-f\left(y_{n}\right)}{x-y_{n}} & =\min \left(\frac{f\left(d_{n}\right)-f(x)}{d_{n}-x}, \frac{f(x)-f\left(c_{n}\right)}{x-c_{n}}\right) \\
& \leqslant \frac{f\left(d_{n}\right)-f(x)+f(x)-f\left(c_{n}\right)}{d_{n}-x+x-c_{n}}<-n .
\end{aligned}
$$

De là et de l'égalité $\lim _{n \rightarrow \infty} x_{n}=\lim _{n \rightarrow \infty} y_{n}=x$ il résulte que

$$
\bar{f}(x)=\infty \quad \text { et } \quad \underline{f}(x)=-\infty .
$$

D'autre part, de $\left(\mathrm{O}_{n}\right)$ et de la définition de $x$ on déduit que $x \in C_{f}$ et, comme $f \in \mathcal{A}$, on a

$$
\bar{f}(x)<\infty \text { ou bien } \underline{f}(x)>-\infty .
$$

Les inégalités (12) et (13) sont incompatibles, et ainsi la démonstration du lemme est achevée.

\section{Structure des ensembles $D_{f}$ et $\Delta_{f}$.}

LeNare 5. Si $A$ et $B$ sont des ensembles quelconques de la classe $F_{\sigma}$, contenus dans $I$, il existe une fonction $f$ definie sur $I$ et satisfaisant aux conditions suivantes:

(2) Nous avons utilisé ici le théorème: Si $b d>0$, on a $\max \left(\frac{a}{b}, \frac{c}{d}\right) \geqslant \frac{a+c}{b+d}$ $\geqslant \min \left(\frac{a}{b}, \frac{c}{d}\right)$.
Si $f(x) \neq 0$, on a $\underset{t \rightarrow x-}{\lim } f(t)\left\langle f(x)>\operatorname{jim}_{t \rightarrow x+} f(t)\right.$ ou

$$
A \backslash B \subset D_{f}=\{x: f(x) \neq 0\} \subset A,
$$

$$
\varlimsup_{t \rightarrow x-} f(t)>f(x)<\varlimsup_{t \rightarrow x+} f(t),
$$

$$
B \backslash A \subset B \cap\{x: f(x)=0\}=B \cap\left\{x: f^{\prime}(x)=0\right\},
$$

$$
|f(x)| \leqslant \frac{1}{2} \text {. }
$$

Démonstration. Soit $a_{n}=2^{-n}$ et $A=\bigcup_{n=0}^{\infty} A_{n}, B=\bigcup_{n=0}^{\infty} B_{n}$, où $A_{0}=B_{0}=0, A_{n}$ et $B_{n}$ étant des ensembles fermés tels que $A_{n} \subset A_{n+1}$ et $B_{n} \subset B_{n}$ pour $n=1,2,3, \ldots$ Désignons par $W$ l'ensemble des nombres rationnels et posons

$$
f(x)=\left\{\begin{array}{ccl}
a_{n} \varrho^{2}\left(x, B_{n}\right) & \text { pour } & x \in\left(A_{n} \backslash A_{n-1}\right) \cap W \text { et } n=1,2,3, \ldots, \\
-a_{n} \varrho^{2}\left(x, B_{n}\right) & \text { pour } & x \in\left(A_{n} \backslash A_{n-1}\right) \backslash W \text { et } n=1,2,3, \ldots, \\
0 & \text { pour } & x \in I \backslash A
\end{array}\right.
$$

où

$$
\varrho(x, E)=\left\{\begin{array}{ccc}
\inf _{y \in E}|x-y| & \text { lorsque } & E \neq 0 \\
1 & \text { lorsque } & E=0 .
\end{array}\right.
$$

La condition (IV) est évidente et la condition (I) sera établie quand nous aurons prouvé quo pour tout point $x$ tel que $f(x) \neq 0$ il existe deux suites $\left\{x_{k}\right\}$ et $\left\{y_{k}\right\}$ satisfaisant aux relations suivantes:

$$
\begin{array}{ll} 
& x_{k}<x<y_{k} \quad \text { pour } \quad k=1,2,3, \ldots, \\
& \lim _{k \rightarrow \infty} f\left(x_{k}\right)<f(x)>\lim _{k \rightarrow \infty} f\left(y_{k}\right) \text { si } \quad x \in W \\
\text { et } & \lim _{k \rightarrow \infty} f\left(x_{k}\right)>f(x)<\lim _{k \rightarrow \infty} f\left(y_{k}\right) \quad \text { si } \quad x \notin W .
\end{array}
$$

D'après la définition de la fonction $f$ et en vertu de l'inégalité $f(x) \neq 0$ on a $x \in A$, donc, pour un $n$ naturel, $x \in A_{n} \backslash A_{n-1}$. Le point $x$ n'est pas un élément de l'ensemble fermé $A_{n-1}$, par conséquent le nombre $\varrho\left(x, A_{n-1}\right)$ est positif. Désignons-le par $\delta$. L'intervalle $(x-\delta, x+\delta) \cap I$ est done contenu dans la sommo des deux ensembles disjoints $A_{n} \backslash A_{n-\mathbf{1}}$ et $I \backslash A_{n}$.

Considérons les deux seuls cas possibles: le premier, où $x$ est l'extrémité d'un intervalle contenu dans $A_{n} \backslash A_{n-1}$, et le second, où $x$ est un point d'accumulation à droite ou à gauche de l'ensemble $I \backslash A_{n}$. Si $x$ est l'extrémité d'un intervallo contenu dans $A_{n} \backslash A_{n-1}$, on peut extraire de cet intervalle deux suites de nombres irrationnels $\left\{t_{k}\right\}$ et de nombres rationnels $\left\{z_{k}\right\}$ convergentes vers $x$. D'où, en tenant compte de la continuité de la fonction $\varrho\left(x, B_{n}\right)$, on obtient

(3) $\lim _{k \rightarrow \infty} f\left(t_{k}\right)=-a_{n} \varrho^{2}\left(x, B_{n}\right)=-f(x)<f(x) \quad$ lorsque $\quad x \in W$, 


$$
\lim _{k \rightarrow \infty} f\left(z_{k}\right)=a_{n} \varrho^{2}\left(x, B_{n}\right)=-f(x)>f(x) \quad \text { lorsque } \quad x \notin W .
$$

Dans le second cas, on peut extraire de l'ensemble $I \backslash A_{n}$ une suite $\left\{\xi_{k}\right\}_{\text {, }}$ convergente vers $x$, de points appartenant a l'intervalle $(0, x)$ ou bien à l'intervalle $(x, 1)$. De la suite infinie et bornée $\left\{f\left(\xi_{k}\right)\right\}$ on peut extraire une suite partielle convergente $\left\{f\left(\xi_{n_{k}}\right)\right\} \equiv\left\{f\left(\eta_{k}\right)\right\}$.

Comme $\eta_{k} \in I \backslash A_{n}=(I \backslash A) \cup \bigcup_{m=n+1}^{\infty}\left(A_{m} \backslash A_{m-1}\right)$, on a $f\left(\eta_{k}\right)=0$ ou bien $f\left(\eta_{k}\right)= \pm a_{m} \varrho^{2}\left(\eta_{k}, B_{m}\right)$, où $m \geqslant n+1$, done toujours

D'où l'on obient

$$
\left|f\left(\eta_{k}\right)\right| \leqslant a_{n+1} \varrho^{2}\left(\eta_{k}, B_{n+1}\right)
$$

(5) $\quad \lim _{k \rightarrow \infty} f\left(\eta_{k}\right) \leqslant \lim _{k \rightarrow \infty}\left|f\left(\eta_{k}\right)\right| \leqslant \lim _{k \rightarrow \infty} a_{n+1} \varrho^{2}\left(\eta_{k}, B_{n+1}\right)=a_{n+1} \varrho^{2}\left(x, B_{n+1}\right)$ $<a_{n} \varrho^{2}\left(x, B_{n}\right)=f(x) \quad$ lorsque $\quad x \in W$,

(6) $\lim _{k \rightarrow \infty} f\left(\eta_{k}\right) \geqslant-\lim _{k \rightarrow \infty}\left|f\left(\eta_{k}\right)\right| \geqslant-\lim _{k \rightarrow \infty} a_{n+1} \varrho^{2}\left(\eta_{k}, B_{n+1}\right)=-a_{n+1} \varrho^{2}\left(x, B_{n+1}\right)$ $>-a_{n} \varrho^{2}\left(x, B_{n}\right)=f(x) \quad$ lorsque $\quad x \notin W$.

Les quatre cas suivants, qui s'excluent mutuellement, peuvent se présenter:

$\left(1^{\circ}\right) \quad x$ est un point intérieur de l'ensemble $A_{n} \backslash A_{n-1}$;

$\left(2^{\circ}\right) \quad x$ est un point d'accumulation da gauche et à droite de l'ensemble $I \backslash A_{n}$;

$\left(3^{\circ}\right) \quad x$ est l'extrémité gauche d'un intervalle contenu dans l'ensemble $A_{n} \backslash A_{n-1}$ et en même temps il est un point d'accumulation à gauche de l'ensemble $I \backslash A_{n}$

$\left(^{\circ}\right) \quad x$ est l'extrémité droite d'un intervalle contenu dans l'ensemble $A_{n} \backslash A_{n-1}$ et en même temps il est un point d'accumulation d droite de l'ensemble $I \backslash \boldsymbol{A}_{n}$.

Dans la suite de cette démonstration nous désignerons $t_{k}$ par $t_{\bar{k}}^{-}$ou $t_{k}^{+}$, suivant que $t_{k}<x$ ou $t_{k}>x$. Des conventions analogues se rapporteront aux points $z_{k}$ et $\eta_{k}$.

Nous définirons maintenant, pour le point considéré $x$, les suites $\left\{x_{k}\right\}$ et $\left\{y_{k}\right\}$. Les définitions de ces suites dépendront des cas $\left(1^{\circ}\right)-\left(4^{\circ}\right)$ et de la rationnalité du point $x$. Dans le cas $\left(1^{\circ}\right)$ nous posons $x_{k}=t_{k}^{-}$et $y_{k}=t_{k}^{+}$ lorsque $x \in W$, ou bien $x_{k}=z_{k}^{-}$et $y_{k}=z_{k}^{+}$lorsque $x \notin W$. Dans le cas $\left(2^{\circ}\right)$ $x_{k}=\eta_{\bar{k}}^{-}$et $y_{k}=\eta_{k}^{+}$, dans le cas $\left(3^{\circ}\right) x_{k}=\eta_{\bar{k}}, y_{k}=t_{k}^{+}$lorsque $x \in W$, ou $y_{k}=z_{k}^{+}$lorsque $x \notin W$. Dans le dernier cas $\left(4^{\circ}\right)$ nous admettons $y_{k}=\eta_{k}^{+}$ et $x_{k}=t_{\bar{k}}$ lorsque $x \in W$, ou $x_{k}=z_{\bar{k}}$ lorsque $x \notin W$. De ces définitions et des formules (3)-(6) il résulte qu'il existe des suites satisfaisant à (1) et (2). La propriété (I) est ainsi établie.
Passons maintenant à la démonstration de la propriété (III). Dans but, considérons $x \in B \cap\{t: f(t)=0\}$. Il existe donc un nombre naturel $n$ tel que $x \in B_{n} \backslash B_{n-1}$. Le point $x$ n'est pas un élément de l'ensemble $A_{n-1}=\bigcup_{m=1}^{n-1}\left(A_{m} \backslash A_{m-1}\right)$. Sinon, on aurait $x \in A_{m} \backslash A_{m-1}$ et $x \notin B_{m}$ pour un $m<n$, et cela donnerait $f(x)= \pm a_{m} \varrho^{2}\left(x, B_{m}\right) \neq 0$, en contradiction avec la condition $f(x)=0$.

De la relation $x \notin A_{n-1}$ il résulte que le nombre $\delta=\varrho\left(x, A_{n-1}\right)$ est positif. Si $h$ est un nombre quelconque satisfaisant à l'inégalité $0<|h|<\delta$, le point $x+h$ n'est pas un élément de l'ensemble $A_{n-1}$. D'où résultent successivement les relations suivantes:

ou bien

$$
x+h \in I \backslash A_{n-1}=(I \backslash A) \cup \bigcup_{m=n}^{\infty}\left(A_{m} \backslash A_{m-1}\right) ; \quad f(x+h)=0
$$

$$
\begin{gathered}
f(x+h)= \pm a_{m} \varrho^{2}\left(x+h, B_{m}\right), \quad \text { où } \quad m \geqslant n ; \\
|f(x+h)| \leqslant a_{n} \varrho^{2}\left(x+h, B_{n}\right) .
\end{gathered}
$$

Le point $x \in B_{n}$, done

$$
\varrho\left(x+h, B_{n}\right) \leqslant \varrho(x+h, x)=|h| \text { et }|f(x+h)| \leqslant a_{n} h^{2} .
$$

On obtient de là pour $0<|h|<\delta$,

$$
\left|\frac{f(x+h)-f(x)}{h}\right|=\frac{|f(x+h)|}{|h|} \leqslant a_{n}|h| .
$$

Nous avons ainsi prouvé que $f^{\prime}(x)=0$ et que $B \cap\{t: f(t)=0\} \subset B \cap$ $\cap\left\{t: f^{\prime}(t)=0\right\}$. D'autre part, si $f^{\prime}(x)=0, f$ est continue au point $x$ et, en vertu de $(\mathrm{I})$; on a $f(x)=0$.

La condition $B \backslash A \subset B \cap\{x: f(x)=0\}$ est évidente.

Reste à établir la propriété (II). Dans ce but, il suffit de prouver les inclusions suivantes:

$$
D_{f} \subset A
$$

puisque l'inclusion

$$
A \backslash B \subset D_{f} \subset\{x: f(x) \neq 0\},
$$

est une conclusion immédiate de (I).

Nous établirons en premier lieu la relation $I \backslash A \subset C_{f}$, équivalente à l'inclusion (7). Soit $x$ un point de l'ensemble $I \backslash A, \varepsilon$ un nombre positif arbitraire et $n$ un nombre naturel tel que $a_{n}<\varepsilon$. L'ensemble $I \backslash A_{n}$ est ouvert (sur $I$ ), il existe done un nombre $\delta>0$ tel que $(x-\delta, x+\delta) \cap I$ $\subset I \backslash A_{n}$. L'ensemble $I \backslash A_{n}=(I \backslash A) \cup \bigcup_{m=n+1}^{\infty}\left(A_{m} \backslash A_{m-1}\right)$. Pour $t \in(x-\delta$, 
$x+\delta) \cap I$ nous avons done $t \epsilon I \backslash A$ ou bien $t \in A_{m} \backslash A_{m-1}$ pour un $m>n$ Les dernières relations et la définition de la fonction $f$ entraînent $f(t)=0$ ou bien $f(t)= \pm a_{m} \varrho^{2}\left(t, B_{m}\right)$, où $m>n$. On a donc toujours $|f(t)|$ $\leqslant a_{n} \varrho^{2}\left(t, \dot{B}_{n}\right)$. D'où l'on obtient pour les mêmes $t$

$$
|f(t)-f(x)|=|f(t)| \leqslant a_{n} \varrho^{2}\left(t, B_{n}\right) \leqslant a_{n}<\varepsilon .
$$

Le point $x$ est donc un point de continuité de la fonction $f$.

L'ensemble $A \backslash B$ est une partio de l'ensemble $\{x: f(x) \neq 0\}$. En effet, si $x \in A \backslash B$, on a pour tout $n \varrho\left(x ; B_{n}\right)>0$, donc $f(x)= \pm a_{n} \varrho^{2}\left(x, B_{n}\right)$ $\neq 0$. De l'inclusion $A \backslash B \subset\{x: f(x) \neq 0\}$ et de (9) résulte la première inclusion (8).

Pour établir la seconde partio de la relation (8) il suffit d'observer que $(A \backslash B) \cap\{x: f(x)=0\}=0$ et que

$$
\begin{aligned}
\{x: f(x)= & 0\} \subset(I \backslash A) \cup[(A \backslash B) \cap\{x: f(x)=0\}] \cup[B \cap\{x: f(x)=0\}] \\
& =(I \backslash A) \cup\left[B \cap\left\{x: f^{\prime}(x)=0\right\}\right] \subset C_{f} \cup C_{f}=C_{f}, \quad \text { c.q.f.d. }
\end{aligned}
$$

Lemare 6. Soient $D \subset I$ un ensemble de la classe $F_{\sigma}, P$ et $Q$ des sousensembles dénombrables de l'intervalle $I$ ne contenant pas les points 0 et 1 . Si les ensembles $D, P$ et $Q$ sont disjoints deux $\dot{a}$ deux et $|D|=1$, il existe une fonction croissante $f$ definie sur $I$ et satisfaisant aux conditions

$$
\begin{gathered}
D_{f}=P \cup Q, \\
\Delta_{f}=D, \\
\left\{x: f^{\prime}(x)=\infty\right\}=I \backslash(D \cup P), \\
\left\{x:\left|f^{-}(x)\right|<\infty\right\} \supset P .
\end{gathered}
$$

Démonstration. Nous définirons la fonction $f$ comme la somme de deux fonctions $g$ et $h$. Définissons d'abord la fonction $g$. Ce sera une fonction de sauts non décroissante statisfaisant aux conditions

$$
\begin{gathered}
D_{g}=P \cup Q, \\
\left\{x: g^{\prime}(x)=0\right\} \supset D, \\
\left\{x: g^{-}(x)=0\right\} \supset P \cup Q .
\end{gathered}
$$

En définissant la fonction $g$ nous distinguerons deux cas $P \cup Q=0$ et $P \cup Q \neq 0$. Si $P \cup Q=0$, nous admettons $g(x)=0$ pour $x \in X$. Dans ce cas les conditions (1)-(3) sont évidemment remplies. D'autre part, si $P \cup Q \neq 0$, nous rangeons les éléments de l'ensemble dénombrable $P \cup Q$ en une suite (finie ou non) $x_{1}, x_{2}, x_{3}, \ldots$, et nous représentons l'ensemble $D \in F_{\sigma}$ comme la somme d'une suite ascendante d'ensembles fermès et non vides $D_{n}$. Nous posons de plus

$$
\alpha=\inf _{n} x_{n}, \quad \alpha_{1}=1 \quad \text { et } \quad \alpha_{n}=\min _{1 \leqslant i<j \leqslant n}\left|x_{i}-x_{j}\right| \quad \text { pour } n=2,3, \ldots
$$

$$
g(x)=\left\{\begin{array}{lll}
0 & \text { pour } & x \in\langle 0, \alpha\rangle, \\
\sum_{x_{n}<x} a_{n} \cdot 2^{-n} \cdot \varrho^{2}\left(x_{n}, D_{n}\right) & \text { pour } & x \in(\alpha, 1\rangle .
\end{array}\right.
$$

La fonction $g$ est non décroissante, puisque $\alpha_{n}>0$.

La condition (1) est remplie, car, les ensembles $P \cup Q$ et $D$ étant disjoints, on a $\varrho\left(x_{n}, D_{n}\right)>0$ pour tout $x_{n}$ et par conséquent

$$
g\left(x_{n}\right)=\sum_{x_{m}<x_{n}} \alpha_{m} \cdot 2^{-m} \cdot \varrho^{2}\left(x_{m}, D_{m}\right)<\sum_{x_{m} \leqslant x_{n}} \alpha_{m} \cdot 2^{-m} \cdot \varrho^{2}\left(x_{m}, D_{m}\right)=\lim _{t \rightarrow x_{n}+} g(t) .
$$

La fonction $g$ admet une dérivée nulle en tout point de l'ensemble $D$. En effet, si $x \in D_{n}$ et $x_{m} \epsilon\langle x, x+h\rangle$, où $0<|h|<\min _{k \leqslant n}\left|x-x_{k}\right|$ on a $m>n$ et $\varrho\left(x_{m}, D_{m}\right) \leqslant \varrho\left(x_{m}, D_{n}\right) \leqslant\left|x_{m}-x\right| \leqslant|\hbar|$. D'où l'on tire

$$
\left|\frac{g(x+h)-g(x)}{h}\right| \leqslant \frac{1}{|h|} \sum_{x_{m} \in\langle x, x+h\rangle} 2^{-m} \cdot \varrho^{2}\left(x_{m}, D_{m}\right) \leqslant \frac{1}{|h|} \sum_{m=1}^{\infty} 2^{-m} h^{2}=|h| .
$$

Cette inégalité prouve que $g^{\prime}(x)=0$. L'inclusion $D_{n} \subset\left\{x: g^{\prime}(x)=0\right\}$ se trouve ainsi établie pour tout $n$ et, par suite, la formule (2) est démontrée.

La condition (3) est évidente lorsque la suite $\left\{x_{n}\right\}$ est finie. Dans le cas contraire, observons que la suite non croissante de nombres $a_{n}$ satisfaisant pour tout $n$ à la condition $0<\alpha_{n} \leqslant 1$ converge vers zéro lorsque $n \rightarrow \infty$. On peut done associer à tout nombre positif $h<1$ un nombre

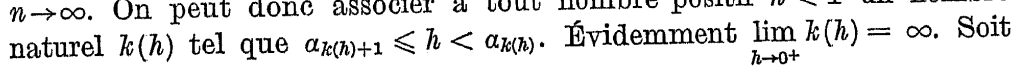
maintenant $p$ un nombre naturel quelconque et $h$ un nombre réel satisfaisant à l'inégalité $0<h<\alpha_{p}$. Cette dernière inégalité et la définition du nombre $k(h)$ prouvent que $k(h) \geqslant p$. Dans l'intervalle $\left\langle x_{p}-h, x_{p}\right\rangle$ $C\left(x_{p}-\alpha_{k(h)}, x_{p}\right)$ il ne peut $\mathrm{y}$ avoir que des points $x_{m}$ pour lesquels $m>k(h)$, donc

$$
\begin{aligned}
\left|\frac{g\left(x_{p}\right)-g\left(x_{p}-h\right)}{h}\right| & =\frac{1}{h} \sum_{x_{m} \in\left\langle x_{p}-h, x_{p}\right)} a_{m} \cdot 2^{-m} \cdot \varrho^{2}\left(x_{m}, D_{m}\right) \\
& \leqslant \frac{1}{\alpha_{k(h)+1}} \sum_{m=k(h)+1}^{\infty} a_{m} \cdot 2^{-m} \leqslant \sum_{m=k(h)+1}^{\infty} 2^{-m}=2^{-k(h)} .
\end{aligned}
$$

De cette limitation on conclut que $g^{-}\left(x_{p}\right)=0$.

Pour l'ensemble $I \backslash(D \cup P)$ de la classe $G_{\delta}$ et de mesure nulle il existe, en vertu d'un théorème de Zahorski [11], une fonction croissante $h$ satisfaisant aux conditions

$$
\begin{gathered}
C_{h}=\Delta_{h}^{*}=I, \\
\left\{x: h^{\prime}(x)=\infty\right\}=I \backslash(D \cup P) .
\end{gathered}
$$


La fonction $f$, étant la somme d'une fonction nou décroissante et d'une fonction croissante $h$, est croissante. Elle admet une dérivé $f^{\prime}(x)=\infty$ partout, où $g^{\prime}(x)=\infty$, ou $h^{\prime}(x)=\infty$, donc, en vertu de $(6)$, on

$$
\left\{x: f^{\prime}(x)=\infty\right\} \supset I \backslash(D \cup P) .
$$

Des relations (5) et (6) il résulte que $\Delta_{h}=D \cup P$, ce qui donne, avec (2) et (3), les inclusions respectives

$$
\Delta_{f} \supset D
$$

$$
\left\{x:\left|f^{-}(x)\right|<\infty\right\} \supset(P \cup Q) \cap\left(D \cup P^{\prime}\right)=P .
$$

La fonction $f$ satisfait à la condition (I), puisque $D_{f}=D_{g}=P \cup Q$ La condition (IV) est anssi remplie, car elle est équivalente à l'inclusion (9). Pour prouver la condition (IX) observons que $\Delta_{f} \subset I \backslash\left(D_{f} \cup\right.$ $\left.\cup\left\{x: f^{\prime}(x)=\infty\right\}\right)$, ce qui donne, avec (I) et (7), $\Delta_{f} \subset I \backslash\{(P \cup Q) \cup$ $\cup[I \backslash(D \cup P)]\}=D$. D'où l'on obtient, en tenant compte de l'inclusion (8), l'égalité (II).

Nous établirons finalement la condition (III). D'après (7), cette condition est équivalente à l'inclusion $\left\{x: f^{\prime}(x)=\infty\right\} \subset I \backslash(D \cup P)$ qui résulte de (II), (IV) et de la relation évidente

$$
\left\{x: f^{\prime}(x)=\infty\right\} \subset I \backslash\left(\Lambda_{f} \cup\left\{x:\left|f^{-}(x)\right|<\infty\right\}\right) .
$$

THEORÈm 1. Si $E, F, G$ et $H$ sont des ensembles quelconques satisfaisant aux conditions

$$
\begin{gathered}
E \subset F \subset G \subset H \subset I, \\
E \in F_{\sigma}, \quad H \in H_{\sigma} \cap G_{\delta}, \\
|H \backslash E|=0, \quad H \backslash F \text { dénombrable, }
\end{gathered}
$$

il existe une fonction $f$ telle que $E=\Delta_{f}, F=O_{f}, G=\Delta_{j}^{*}$.

Démonstration. Nous définirons la fonction $f$ comme la somme de six fonctions tout au plus $\varphi_{0}, \varphi_{1}, \psi_{0}, \psi_{1}, \varphi$ et $\psi$, dont les quatre premières seront définies par les formules

$$
\begin{aligned}
& \varphi_{k}(x)=\left\{\begin{array}{lll}
0 & \text { pour } & x=k, \\
2 \sin \frac{\pi}{x-k} & \text { pour } & x \neq k
\end{array}\right. \\
& \psi_{k}(x)=\left\{\begin{array}{lll}
0 & \text { pour } & x \neq k, \\
(-1)^{1+k} & \text { pour } & x=k .
\end{array}\right.
\end{aligned}
$$

Pour définir la fonction suivante nous profiterons du lemmo 5 , en vertu duquel il existe pour les ensembles $A=I \backslash H$ et $B=H$ une fonc-

tion $\varphi$ satisfaisant aux conditions (I)-(IV) de ce lemme. Puisque les ensembles $A$ et $B$ sont disjoints et que $A \cup B=I$, ces conditions sont équivalentes aux suivantes:

$$
\begin{aligned}
& D_{\varphi}=\{x: \varphi(x) \neq 0\}=I \backslash H ; \\
& \Delta_{\varphi}=\left\{x: \varphi^{\prime}(x)=0\right\}=\{x: \varphi(x)=0\}=H ;
\end{aligned}
$$

si $x \in D_{\varphi}$, on a

$$
\varliminf_{t \rightarrow x-} \varphi(t)<\varphi(x)>\varliminf_{t \rightarrow x+} \varphi(t) \quad \text { ou bien } \quad \varlimsup_{t \rightarrow x-} \varphi(t)>\varphi(x)<\varlimsup_{t \rightarrow x+} \varphi(t) ;
$$

$$
|\varphi(x)| \leqslant \frac{1}{2} \text { pour } x \in I .
$$

Nous définirons mainetnant la fonction $\psi$. Dans ce but, nous profiterons du lemme 6, en vertu duquel il existe, pour les ensembles $D=I \backslash(H \backslash E), P=(H \backslash G) \cap(0,1)$ et $Q=\left(G \backslash F^{\prime}\right) \cap(0,1)$ une fonction croissante $\psi$ safisfaisant aux conditions

(7) $D_{\varphi}=P \cup Q=\left(H \backslash F^{\prime}\right) \cap(0,1)=\left(H \backslash F^{\prime}\right) \backslash\left(P_{1} \cup Q_{1}\right)$,

$$
\text { où } \quad P_{1}=(H \backslash G) \backslash(0,1), \quad Q_{1}=(G \backslash F) \backslash(0,1) \text {; }
$$

$\left\{x: \psi^{\prime}(x)=\infty\right\}=I \backslash(D \cup P)=(G \backslash E) \cup P_{1}$

$$
\text { (10) }\left\{x:\left|\psi^{-}(x)\right|<\infty\right\} \supset P=(H \backslash G) \cap(0,1)=(H \backslash G) \backslash P_{1} \text {. }
$$

Posons $\chi(x)=\varphi(x)+\psi(x)$. Nous démontrerons plus loin que la fonction ainsi définie $\chi$ satisfait aux conditions suivantes:

$$
\begin{gathered}
C_{x}=F \cup P_{1} \cup Q_{1}, \\
\Delta_{x}=E,
\end{gathered}
$$

$$
G \cup P_{1} \subset \Delta_{x}^{*} \subset G \cup R_{1}, \quad \text { où } \quad R_{1}=(I \backslash G) \backslash(0,1) .
$$

Des égalités. (3) et (7) il résulte que

done

$$
D_{\varphi} \cap D_{\varphi} \subset(I \backslash H) \cap(H \backslash F)=0,
$$

$$
D_{x}=D_{\varphi} \cup D_{\varphi}=(I \backslash H) \cup\left[(H \backslash F) \backslash\left(P_{1} \cup Q_{1}\right)\right]=(I \backslash F) \backslash\left(P_{1} \cup Q_{1}\right) .
$$

Cette dernière relation est équivalente à la condition (11). L'égalité (12) est aussi vérifiée. En effet, les fonctions $\varphi$ et $\psi$ sont dérivables aux points de l'ensemble $E$ (voir (4) et (8)), donc

$$
E \subset \Delta_{x} \text {. }
$$

D'autre part, aux points de l'ensemble $(G \backslash E) \cup P_{1}$ qui est partie de l'ensemble $H$, la fonction $\varphi$ admet une dérivée finie et la fonction $\psi$ une dérivée égale à $+\infty$, donc

$$
(G \backslash E) \cup P_{1} \subset\left\{x: \chi^{\prime}(x)=\infty\right\} .
$$


D'où, en tenant compte de l'inclusion évidente $\Delta_{x} \subset O_{x} \backslash\left\{x: \chi^{\prime}(x)=\infty\right\}$ il résulte que $\Delta_{x} \subset\left(F \cup P_{1} \cup Q_{1}\right) \backslash\left[(G \backslash E) \cup P_{1}\right]=E$; avec (14) cela éta. blit l'égalité (12).

La première inclusion (13) résulte immédiatement de la formule (12) et (15) et de l'inclusion évidente $\Delta_{\chi} \cup\left\{x: \chi^{\prime}(x)=\infty\right\} \subset \Delta_{x}^{*}$. Reste à établir la seconde inclusion (13). Dans ce but, considérons $x \in(H \backslash G) \cap(0,1)$. Arec cette hypothèse on a $\varphi^{\prime}(x)=0$, tandis que $\psi^{-}(x)$ existe et est finie. La dérivée $\psi^{+}(x)=\infty$, puisque $x$ est, en vertu de $(7)$ et $(\mathbf{1 0})$, point de dsicontinuité à droite de la fonction croissante $\psi$. Des deux dernières conclusions il résulte que $\chi^{+}(x)=\infty$ et $\chi^{-}(x) \neq \pm \infty$. Nous avons ainsi établi l'inclusion

$$
(H \backslash G) \cap(0,1) \subset I \backslash \Delta_{x}^{*} .
$$

Des formules (3) et (5) il résulte que si $x \in(0,1) \cap D_{p}$, on a $\underline{\varphi(x)=-\infty}$, $\bar{\varphi}(x)=\infty$; les seuls points de l'ensemble $D_{\varphi}=I \backslash H$, où peüt exister la dérivée $\varphi^{\prime}(x)$ sont donc 0 et 1 (si ces points appartiennent à l'ensemble $I \backslash H$ ). La fonction $\psi$ admettant aux points de l'ensemble $I \backslash H$ une dérivée finie, on a donc

$$
(I \backslash H) \cap(0,1) \subset I \backslash \Delta_{x}^{*} .
$$

Les relations $(16)$ et $(17)$ donnent $(I \backslash G) \cap(0,1) \subset I \backslash \Delta_{x}^{*}$. Cette inclusion est équivalente à la seconde inclusion (13).

Nous définirons maintenant la fonction $f$ en posant pour $x \in I$

$$
f(x)=\chi(x)+\sum_{k=0}^{1}\left[a_{k} \varphi_{k}(x)+b_{k} \psi_{k}(x)\right]
$$

où l'on a, pour $k=0$ ou $k=1$,

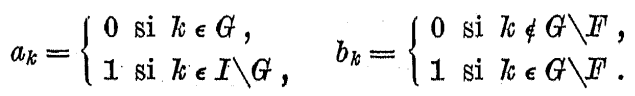

Pour démontrer les égalités

$$
\Delta_{f}=E, \quad O_{f}=F \quad \text { ot } \quad \Delta_{f}^{*}=G
$$

nous nous appuierons sur les conclusions suivantes:

(A) Si $g$ est une fonction definie dans l'intervalle $\langle 0,1\rangle$ satisfaisant pour $k=0$ ou $k=1$ aux conditions
(20)

$$
\left|g(k)-\varliminf_{x \rightarrow c} g(x)\right| \leqslant 1 \quad \text { et } \quad\left|g(k)-\varlimsup_{x \rightarrow l c} g(x)\right| \leqslant 1
$$

et si $h=g+\varphi_{k}$, on $a$

$$
\Delta_{h}=\Delta_{g} \backslash\langle k\rangle, \quad C_{h}=C_{g} \backslash\langle k\rangle \quad \text { et } \quad \Delta_{h}^{*}=\Delta_{0}^{*} \backslash\langle k\rangle, \quad \text { oì } \quad\langle k\rangle=\langle k, k\rangle \text {. }
$$

(B) Si k (égal à 0 ou 1 ) est point de continuité de la fonction $g$ définie dans l'intervalle $\langle 0,1\rangle$, la fonction $h=g+\psi_{k}$ vérifie les égalités $\Delta_{h}=\Delta_{g} \backslash\langle k\rangle, C_{h}=C_{g} \backslash\langle k\rangle$ et $\Delta_{h}^{*}=\Delta_{g}^{*} \cup\langle k\rangle$.

(C) Si la fonction $p$ définie. dans l'intervalle $\langle 0,1\rangle$ est continue au point $k(k=0$ ou $k=1$ ), la fonction $g=\varphi+p$ satisfait aux conditions (20). En particulier, la foncton $\chi$ satisfait aux conditions (20).

La conclusion (A) est vraie. En effet, de la formule

$$
h(x)=\left\{\begin{array}{lll}
g(x) & \text { pour } & x=k, \\
g(x)+2 \sin \frac{1}{x-k} & \text { pour } & x \in(k, 1-k\rangle=\langle 1-k, k),
\end{array}\right.
$$

il résulte que

$$
\begin{aligned}
\varliminf_{x \rightarrow k} h(x) & \leqslant \varlimsup_{x \rightarrow k} g(x)+2 \lim _{x \rightarrow k} \sin \frac{1}{x-k} \\
& \leqslant g(k)+1-2<h(k)<g(k)-1+2 \\
& \leqslant \varliminf_{x \rightarrow k} g(x)+2 \varlimsup_{x \rightarrow k} \sin \frac{1}{x-k} \leqslant \varlimsup_{x \rightarrow k} h(x) .
\end{aligned}
$$

D'où, en tenant compte du fait que $k$ est extrémité de l'intervalle $\langle 0,1\rangle$, on conclut que $k \notin C_{h}$ et $k \notin \Delta_{h}^{*}$.

La conclusion (B) résulte immédiatement de ce que la fonction $g$ est continue au point $k$ et de la formule

$$
h(x)=\left\{\begin{array}{lll}
g(x) & \text { pour } \quad x \in(k, 1-k\rangle, \\
g(x)+(-1)^{1+k} & \text { pour } \quad x=k .
\end{array}\right.
$$

De même, la conclusion (C) résulte immédiatement de l'inégalité (6).

Nous étudierons maintenant la formule (18) définissant la fonction $f$; les seuls cas possibles et s'excluant mutuellement sont les suivants: $1^{\circ} 0 \in F$ et $1 \in F ; 2^{\circ} k \in F^{\prime}(k=0$ ou $k=1)$ et $1-k \in G \backslash F ; 3^{\circ} k \in F$. et $1-k \in I \backslash G ; 4^{\circ} 0 \in G \backslash F$ et $1 \in G \backslash F ; 5^{\circ} k \epsilon G \backslash F$ et $1-k \in I \backslash G ; 6^{\circ} 0 \in I \backslash G$ et $1 \in I \backslash G$.

Dans le cas $1^{\circ}$ la fonction $f=\chi$ et les ensembles $P_{1}, Q_{1}$ et $R_{1}$ sont vides. D'où, en tenant compte des formules (11)-(13), on tire les égalités (19).

Dans le cas $2^{\circ}$ on a $P_{1}=R_{1}=0, Q_{1}=\langle 1-k\rangle$ et $f=\chi+\psi_{1-k}$. Cette dernière égalité et la conclusion (B) (pour $g=\chi$ et $h=f$ ) donnent

$\Delta_{f}=\Delta_{x} \backslash\langle 1-k\rangle=E \backslash Q_{1}=E, \quad C_{f}=C_{\chi} \backslash\langle 1-k\rangle=\left(F \cup P_{1} \cup Q_{1}\right) \backslash Q_{1}=F$ et $\quad \Delta_{f}^{*}=\Delta_{x} \cup\langle 1-k\rangle=\Delta_{x}^{*} \cup Q_{1} \subset G \cup R_{1} \cup Q_{1}=G$.

L'inclusion $\Delta_{f}^{*} \subset G$ établie précédemment, rapprochée de la première inclusion (13), donne l'égalité $\Delta_{f}^{*}=G$. 
Si $k \in F^{\prime}$ et $1-k \in I \backslash G\left(\operatorname{cas} 3^{\circ}\right)$, on a $f=\chi+p_{1-k}$. La fonction $\chi$ satisfait à la condition (20). Avec (A) cela donne

$\Delta_{f}=\Delta_{x} \backslash\langle 1-7\rangle=\Delta_{x}=E, \quad C_{f}=C_{x} \backslash\langle 1-k\rangle \quad$ et $\quad \Delta_{f}^{*}=\Delta_{x}^{*} \backslash\langle 1-k\rangle$.

Ces formules, avec les égalités $Q_{1}=0$ et $R_{1}=\langle 1-k\rangle$ et les relations (11) et (13), donnent les égalités (19).

Dans le cas $4^{\circ}$ on a $f=\chi+\psi_{0}+\psi_{1}, P_{1}=R_{1}=0$ et $Q_{1}=\langle 0\rangle \cup\langle 1\rangle$. La fonction $\chi$ est continue au point 0 et la fonction $g=\chi+\psi_{0}$ est continue au point 1; en appliquant done derx fois la conclusion (B) on obtient $\Delta_{g}=\Delta_{x} \backslash\langle 0\rangle, \quad C_{g}=O_{x} \backslash\langle 0\rangle$ et $\Delta_{g}^{*}=\Delta_{x}^{*} \cup\langle 0\rangle$ ainsi que $\Delta_{f}=\Delta_{g} \backslash\langle 1\rangle$, $O_{f}=C_{d} \backslash\langle 1\rangle$ et $\Delta_{f}^{*}=\Delta_{v}^{*} \cup\langle 1\rangle$. D'où, en tenanti compte des relations (11)-(13), résultent Iess égalités $\Delta_{f}=\Delta_{\alpha} \backslash(\langle 0\rangle \cup\langle 1\rangle)=E \backslash \mathbb{Q}_{1}=E$ ot $C_{f}=C_{x} \backslash Q_{1}=F$ ainsi que l'inclusion $\Delta_{y}^{*}=\Lambda_{x}^{*} \cup Q_{1} \subset G \cup R_{1} \cup Q_{1}=G$; rapprochée de l'inclusion $\Delta_{\gamma}^{*} \supset \Delta_{x}^{*} \supset G$ celle ci donne l'égalité $\Delta_{f}^{*}=G$.

Considérons maintenant le cas $5^{\circ}$, pour lequel $f=x+\varphi_{1-k}+\psi_{k}$, $Q_{1}=\langle k\rangle$ et $R_{1}=\langle 1-k\rangle$. La fonction $g=\chi+\varphi_{1-k}$ vérifie les égalités $\Delta_{g}=E, O_{g}=O_{x} \backslash\langle 1-k\rangle$ et $\Delta_{v}^{*}=\Delta_{x}^{*} \backslash\langle 1-k\rangle$. Cela résulte du raisonnement fait dans le cas $3^{\circ}$. La fonction $g=\chi+p_{1-k}$ est aussi continue au point $k$, puisque $k \in Q_{1} \subset O_{x}$. En vertu de (B) on a done $\Delta_{f}=\Delta_{g} \backslash\langle k\rangle=E$, $O_{f}=C_{g} \backslash\langle k\rangle=O_{x} \backslash\left(Q_{1} \cup R_{1}\right)$ et $\Delta_{f}^{*}=\Delta_{f}^{*} \cup\langle k\rangle=\left(A_{x}^{*} \backslash\langle 1-7\rangle\right) \cup\langle k\rangle$. Ces dernières relations, avec (11) et (13), entrainent les égalités (19).

Le dernier cas $6^{\circ}$ se présente lorsque 0 et 1 sont points de l'senemble $I \backslash G$. Alors $f=\chi+\varphi_{0}+\varphi_{1}, R_{1}=\langle 0\rangle \cup\langle 1\rangle$ et $Q_{1}=0$. En posant dans le raisonnement du cas $3^{\circ} k=1$, on obtient pour la fonction $g=\chi+\varphi_{0}$ les égalités suivantes: $\Delta_{g}=\mathbb{E}, C_{g}=C_{x} \backslash\langle 0\rangle$ et $\Delta_{y}^{*}=\Delta_{\chi}^{*} \backslash\langle 0\rangle$. La fonction $g=\varphi+\left(\psi+\varphi_{0}\right)$ satisfait à la condition $(20)$ pour $k=1$. Cela résulte de la conclusion (C) et de la continuité des fonctions $\psi$ et $\varphi_{0}$ au point 1. En appliquant maintenant à la fonction $f=g+\varphi_{1}$ la conclusion (A), on obtient les égalités $\Delta_{f}=\Delta_{g} \backslash\langle 1\rangle=E, \quad O_{f}=O_{g} \backslash\langle 1\rangle=O_{z} \backslash R_{1}$ et $\Delta_{f}^{*}$ $=\Delta_{g}^{*} \backslash\langle 1\rangle=\Delta_{x}^{*} \backslash R_{1}$, d'où résultent les égalités (19), c.q.f.d.

Lemas 7. Si l'ensemble $B \in F_{\sigma}, C \in G_{\delta}$ et $C \subset B C I$, il existe un ensemble $K \in F_{\sigma} \cap G_{\delta}$ tel que $O \subset K \subset B$.

Démonstration. Pour les ensembles $A=I \backslash O$ et $B$ il existe, en vertu du lemme 5 , une fonction $f$ satisfaisant aux conditions (I)-(IV) de ce lemme. De l'égalité $A \cup B=I$ et des conditions (II) et (III) il résulte que $\Delta_{f} \subset C_{f}=\{x: f(x)=0\}=\{x: f(x)=0\} \cap B \subset \Delta_{f}$. D'où l'on obtient l'égalité $\Delta_{f}=C_{f}$. L'ensemble $C_{f}$ est donc égal à l'ensemble de tous les points $x$, où la fonction $f$ a toutes ses dérivées de Dini finies, il est donc un $F_{\sigma}$ (v. [10]). Nous avons ainsi prouvé que l'ensemble $K=C_{f} \in F_{\sigma} \cap G_{\delta}$. (La méme conclusion résulte du théorème de Kronrod cité dans l'introduction). Pour achever la démonstration, il suffit d'observer que $C=B \backslash A \subset \Delta_{f}=O_{f}=I \backslash D_{f} \subset I \backslash(A \backslash B)=B$.
Coroltatre 1. Si $f \in \mathcal{A}$, on a $D_{f}=K \cup P, B_{f}=K \backslash Q$, où $K \in F_{\sigma} \cap G_{\delta}$ et $P$ et $Q$ sont des ensembles dénombrables.

Démonstration. En vertu du lemme 4 l'ensemble $B_{f}$ est un $G_{\delta}$ et, en vertu du lemme 3 , on a $B_{f} \subset D_{f}$. Puisque $D_{f} \in F_{\sigma}$, il existe, en vertu du lemme 6 , un ensemble $K$ tel que $B_{f} \subset K \subset D_{f}$ et $K \in F_{\sigma} \cap G_{\delta}$. D'après le lemme 3 , les ensembles $P=D_{f} \backslash K$ et $Q=K \backslash B_{f}$, étant sous-ensembles de l'ensemble dénombrable $D_{f} \backslash B_{f}$, sont dénombrables. Évidemment $D_{f}=K \cup P, B_{f}=K \backslash Q$, c.q.f.d.

Tmóorìms 2. Pour que l'ensemble A soit l'ensemble des points de discontinuité d'une fonction $f$ admettant une dérivée (finie ou infinie) en tout point de continuite, il faut et il suffit que $A=K \cup P$, où $K \in F_{\sigma} \cap G_{\delta}$ et $P$ est un ensemble dénombrable.

THÉoRìme 2'. Pour que l'ensemble A soit l'ensemble des points de discontinuité d'une fonction $f \in \mathcal{A}$, il faut et il suffit que $A=K \cup P$, ò̀ $K \in F_{\sigma} \cap G_{\delta}$, et $P$ est un ensemble dénombrable.

Démonstration des théor èmes 2 et $2^{\prime}$. Puisque chaque fonction $f$ satisfaisant à l'inclusion $\Delta_{f}^{*} \supset O_{f}$ est une fonction de l'ensemble $\mathcal{A}$, il suffit, pour établir les théorèmes 2 et 2 , de démontrer les propositions suivantes:

(A) Si $A=D_{f}$ et $f \in \mathcal{H}$, on a $A=K \cup P$, où $K \in F_{\sigma} \cap G_{\delta}$ et $P$ est un ensemble dénombrable.

(B) Si $A=K \cup P$, oì $K \in F_{\sigma} \cap G_{\delta}$ et $P$ est un ensemble dénombrable, il existe une fonction $f$ telle que $\Delta_{f}^{*} \supset O_{f}$ et $A=D_{f}$.

La proposition (A) résulte du corollaire 1. Il n'y a donc qu'à prouver la proposition (B). Dans ce but supposons que $A$ soit la somme de deux ensembles, dont le premier $K$ est un $F_{\sigma}$ et en même temps un $G_{s}$, le second $P$ est dénombrable. On peut définir les ensembles $E, F, G$ et $H$ de telle manière que les hypothèses du théorème 1 soient vérifiées. Il suffit, en effet, de poser $F=G=I \backslash A, H=I \backslash K$ et de prendre pour $E$ un sousensemble quelconque $F_{\sigma}$ de l'ensemble $I \backslash A$ tel que $|E|=|I \backslash A|$. Il existe alors une fonction $f$ telle que $\Delta_{f}^{*}=O_{f}=I \backslash A$ et $D_{f}=I \backslash F=A$, c.q.f.d.

THÉorìme 3. Pour que l'ensemble $E$ soit l'ensemble des points de dérivabilité d'une fonction $f$ admettant une dérivée (non nécessairement finie) en tout point de continuité, il faut et il suffit que $E=H \backslash C$, ò̀ $H \in F_{\sigma} \cap G_{\delta}$ et $O \subset A$ est un $G_{\delta}$ de mesure nulle.

Démonstiation. Soit $\Delta_{f}=\mathbb{E}$ et soit $f$ une fonction admettant une dérivée en tout point de continuité. Pour une telle fonction l'ensemble $C_{f}$ est, en vertu du théorème 2 , égal à $H \backslash P$, où l'ensemble $H$ est en même temps un $F_{\sigma}$ et un $G_{\delta}$ et l'ensemble $P$ est dénombrable.

Nous profiterons maintenant du théorème suivant (v. Ward [7] et [8]): Toute fonction $\varphi$ admet une dérivée finie en presque tout point de l'ensemble $S_{\varphi}=\{x: \varphi(x)>-\infty$ ou $\bar{\varphi}(x)<\infty\}$. 
De ce théorème résulte que l'ensemble $\left\{x:\left|f^{\prime}(x)\right|=\infty\right\}$, étant sousensemble de l'ensemble $S_{f} \backslash \Delta_{f}$, est de mesure nulle done $|H \backslash E|=\left|C_{f} \backslash E\right|$ $=\left|\Delta_{f}^{*} \backslash \Delta_{f}\right|=\left|\left\{x:\left|f^{\prime}(x)\right|=\infty\right\}\right|=0$. On sait anssi (v. [10]) que pour toute fonction $f$ l'ensemble des points, où toutes les dérivées de Dini sont finies, est un $F_{\sigma}$. Sous les hypothèses de notre théorème cet ensemble est identique à l'ensemble $\Delta_{f}=E$, donc $E \in F_{\sigma}$. Il en résulte que l'ensemble $C=H \backslash E \in G_{\delta}$. Nous avons ainsi prouvé que $E=H \backslash C$, où $H \in F_{\sigma} \cap G_{\delta}$ et $C$ est un $G_{\delta}$ de mesure nulle.

Réciproquement supposons que $E=H \backslash O$, où les ensembles $H$ et $C$ satisfont aux mêmes conditions que précédemment. Posons dans le théorème $1 F=G=H$. Il existe donc une fonction $f$ telle que $\Delta_{f}=E$ et $C_{f}=F=G=\Delta_{f}^{*}$, e.q.f.d.

Remarque 1. En s'appuyant sur les théorèmes 1,2 et 3 on démontre aisément le théorème suivant:

Pour que les ensembles $E$ et $F$ soient respectivement àgaux aux ensembles $\Delta_{f}$ et $C_{f}$ d'une fonction $f$ admettant une dérivée en tout point de continuité, il faut et il suffit qu'il existe un ensemble $H$ tel que

$$
E \subset F \subset H \subset I \quad \text { et } \quad|H \backslash E|=0,
$$

$\left(2^{\circ}\right) \quad \forall \in F_{\sigma}, \quad H \in F_{\sigma} \cap G_{\delta}, \quad H \backslash F^{\prime}$ dénombrable.

Remarque 2. Si $f \in \mathfrak{A}$, il existe une dérivée finie en presque tout point de l'ensemble $C_{f}$. Cela résulte du théorème de M. Ward cité dans la démonstration du théorème 3 . La classe des fonctions $f$ telles que $\left|C_{f} \backslash \Delta_{f}\right|=0$ est effectivement plus large que la classe $t$. L'ensemble des points de discontinuité d'une fonction de cette classe peut être un $\mathbb{F}_{\sigma}$ tout à fait arbitraire. En effet, si $A \in F_{\sigma}$ et $\mathbb{E}$ désigne un sous-ensemble quelconque $F_{\sigma}$ de l'ensemble $I \backslash A$ ayant même mesure que $I \backslash A$, il existe pour les ensembles $A$ et $B=E$, en vertu du lemme 5 , une fonction $f$ telle que $D_{f}=A \backslash E=A,\left\{x: f^{\prime}(x)=0\right\}=E \backslash A=E$. On a donc $O_{f} \backslash \Delta_{f}$ $C(I \backslash A) \backslash E$ et $\left|O_{f} \backslash \Delta_{f}\right| \leqslant|(I \backslash A) \backslash E|=0$.

Remarque 3. En choisissant convenablement, dans les lemmes 5 et 6 , respectivement les ensembles $A$ et $B$, ainsi que $D, I^{P}$ et $Q$, on peut obtenir certains résultats connus. Par exemple, lorsque les ensembles $A$ et $B$ sont disjoints et $A \cup B=I$, le lemme 5 fournit la preuve quo la condition donnée dans le théorème de Kronrod, cité dans l'introduction, est suffisante. De même, lorsque $P=0$ et $Q$ est un ensemble dénombrable quelconque, la fonction $f$ du lemme 6 est non décroissante, admet partout une dérivée et l'ensemble de ses points de discontinuité $D_{f}=Q$. Des exemples de telles fonctions ont été donnés par Garg [1] et Marcus [5].

Considérons encore l'exemple de la fonction $f$ que l'on obtient du lemme 6 lorsque $P=0, Q$ est un ensemble dénombrable quelconque
Sur la structure de l'ensemble des points de discontinuité des fonctions

et $I \backslash D$ un $G_{\delta}$ quelconque de mesure nulle contenant l'ensemble $Q$. Alors la fonction $f$ a les propriétés suivantes:

$$
\begin{gathered}
\text { elle est non décroissante sur } I, \\
\\
D_{f}=Q, \\
\Delta_{f}^{*}=I, \\
\Delta_{f}=\Delta \quad \text { et } \quad\left\{x: f^{\prime}(x)=\infty\right\}=I \backslash D .
\end{gathered}
$$

L'existence d'une telle fonction $f$ a été démontrée par Lipiński dans [4].

Remarque 4. Dans la classe des fonctions $f$ satisfaisant à la condition $\Delta_{f}^{*} \supset C_{f}$, l'ensemble $\Delta_{f}^{*}$ est, en vertu du corollaire 1 , de la forme $(H \backslash P) \cup Q$, où $P$ et $Q$ sont des ensembles dénombrables et $H \in F_{\sigma}^{\prime} \cap G_{\delta}$. Il résulte du théorème 1 que cet ensemble peut être de la forme $H \backslash P$, c'est-à-dire que si les ensembles $H \in F_{\sigma} \cap G$ * sont quelconques et $P$ dénombrable, il existe une fonction $f$ telle que $\Delta_{f}^{*}=H \backslash P \supset C_{f}$. Ce fait donne lieu à poser la question suivante: quelle est la structure de l'ensemble $\Delta_{f}^{*}$ pour une fonction $f$ qui admet une dérivée finie ou infinie en tout point de continuité?

\section{Travaux cités}

[1] M. K. Garg, On the derivability of functions discontinuous at a dense set, Revue de Math. pures et appl., Acad. R.P.R. 7 (1962), pp. 175-179.

[2] A. S. Kronrod, Sur la structure de l'ensemble des points de discontinuité d'une fonction dérivable en ses points de continuité, Izv. Akad. Nauk (1939), pp. 569-578.

[3] K. Kuratowski, Topologie (volume 1), Monografie Matematyczne t. XX, Warszawa 1952

[4] J. S. Lipiński, Sur quelques problèmes de S. Marcus relatifs à la dérivée d'une fonction monotone, Revue de Math. pures et appl. Acad. R.P.R. 8 (1963).

[5] S. Marcus, Точки разрыва и точки в которых производная является бесконечной, Revue de Math. pures et appl., Acad. R.P.R. 7 (1962), pp. 309-318.

[6] W. Sierpiński, Wstep do teorii funkcji zmiennej rzeczywistej, Lwów-Warszawa 1932 .

[7] A. J. Ward, On the differentiation of the additive functions of rectangles, Fund. Math. 26 (1936), pp. 167-182.

[8] - On the derivation of additive functions of intervals in m-dimensional space, Fund. Math. 28 (1937), pp. 265-279.

[9] W. H. Young, La symétrie de structure des fonctions de variables réelles, Bulletin des sciences math. (2) 52 (1928), pp. 265-280.

[10] - On the infinite derivatives of a function of a single real variable, Arkiv för Mat. Astr. och Fysilk I (1903), pp. 201-204.

[11] Z. Zahorski, Über die Ifenge der Punkte in welchen die Ableitung unendlich ist, Tohoku Math. Journ. 48 (1941), pp. 321-330.

UNIVERSITE DE EODZ

Regu par la Rédaction le 1. 1. 1966 